\title{
Supplier Relationship Management im Krankenhaus
}

Strukturelle Veränderungen des Gesundheitswesens und eine zunehmende Marktdynamik führen dazu, dass sich Krankenhäuser verstärkt um Prozessoptimierung und Kosteneinsparungen bemühen müssen. Eine aktive und differenzierte Gestaltung der Beziehungen zu Lieferanten gewinnt dabei immer mehr an Bedeutung. Die Fallstudie aus dem Universitätsspital Zürich zeigt, dass durch Einführung verschiedener Instrumente des Supplier Relationship Management (SRM) erhebliche Vorteile entstehen können. Aus organisatorischer Sicht kann durch Gründung eines Einkaufsverbunds Markttransparenz geschaffen werden, was später bei Verhandlungen über Preise und Konditionen genutzt werden kann. Aus technischer Sicht kann durch Einführung verschiedener E-ProcurementTools nicht nur die Effizienz gesteigert, sondern auch die Qualität der Informationen erhöht werden. Dabei profitiert sowohl die Einkaufsabteilung als auch das Pflegepersonal.

\section{Inhaltsübersicht}

1 Bedeutung der Lieferantenbeziehung im Gesundheitswesen

2 SRM in Krankenhäusern

2.1 Strategisches Management der Lieferantenbeziehung (Sourcing)

2.2 Operative Beschaffungsaufgaben (Procurement)

2.3 Monitoring und Controlling der Lieferantenbeziehung

3 SRM im Universitätsspital Zürich - eine Fallstudie

3.1 Strategisches Sourcing: Die Gründung des Einkaufsverbunds Symbia

3.2 Operatives Procurement: Die Nutzung von Desktop Purchasing

3.3 Nutzen für das Universitätsspital Zürich
4 Mehr SRM im Gesundheitswesen

5 Literatur

\section{Bedeutung der Lieferanten- beziehung im Gesundheitswesen}

Die weltweite Ausdehnung der Märkte (Globalisierung), die Individualisierung von Produkten und Dienstleistungen (fragmentierte Märkte), der Druck stetiger Innovationen (steigende Innovations- und Wissensintensität) sowie immer kostengünstigere Möglichkeiten der Datenübertragung und -verarbeitung (technologische Entwicklungen) haben in wettbewerbsintensiven Branchen wie beispielsweise der Automobil- oder Elektroindustrie dazu geführt, dass sich eine ausgeprägte Vernetzung mit hoher Arbeitsteilung und optimierter Prozessorganisation zwischen den einzelnen Marktteilnehmern entwickelt hat (vgl. [Riemer \& Klein 2002, S. 6]). Diese fundamentalen Marktveränderungen haben auch zu einem Paradigmenwechsel in der Beschaffung geführt: "Stand einst das Ziel der Kosteneinsparung im Vordergrund, werden heute vom Einkauf auch Erlössteigerungen sowie die Akquisition von Innovationen auf den Zuliefermärkten verlangt “ [Stölzle \& Heusler 2003, S. 169]. Die Rolle des Lieferanten, der früher im Rahmen von Preisverhandlungen als Gegner betrachtet wurde, hat sich zu der eines Wertschöpfungspartners hin entwickelt, der in die Beschaffungsprozesse integriert werden soll. Demzufolge gewinnt die aktive und differenzierte Gestaltung der Beziehungen zu Lieferanten immer mehr an Bedeutung. Gerade mit diesem Teilbereich des Partner Relationship Management (nämlich Fokus auf Beziehungen mit externen Lieferanten) beschäftigt sich das Supplier Relationship Management (SRM). Nebst der Abwicklung tradi- 
tioneller operativer Tätigkeiten des Einkaufs (z. B. E-Procurement) verbindet SRM organisatorische und insbesondere auch strategische Aspekte der Beschaffung.

Im Gesundheitswesen kristallisieren sich erste Anzeichen für ähnliche Marktveränderungen heraus. Die Akteure sind in Bewegung: Die Schaffung neuer rechtlicher Formen für öffentliche Krankenhäuser und Kliniken sowie vermehrte Zusammenschlüsse und Konzentrationen zeigen dies. Maßnahmen zur Steigerung von Effizienz und Effektivität treten je länger, je mehr in den Vordergrund (vgl. [Albrecht \& Töpfer 2006, S. 3 f.]). Neuere Entwicklungen wie beispielsweise die Einführung der "Diagnosis Related Groups (DRG)«-Tarifierung führen dazu, dass zeit- und kosteneinsparende Arbeitsabläufe im Krankenhaus deutlich mehr Beachtung erfahren werden (vgl. [Falk \& Da-Cruz 2005; Flintrop 2006].

Damit ein Krankenhaus im Sinne der DRGTarifierung rentabel geführt werden kann, müssen künftig auch die Beziehungen zu den externen Partnern besser in die eigenen Strukturen integriert werden. Dabei kommt der Lieferantenbeziehung eine gewichtige Rolle zu. Die Aspekte für deren Gestaltung sind vielfältig:

Steigender Sachkostenanteil an den Gesamtkosten: Obwohl die Personalkosten immer noch den Löwenanteil an den Gesamtkosten eines Krankenhauses ausmachen (in Deutschland im Jahr 2005 ca. 63.67\%), steigen die Kosten für Sachmittel kontinuierlich an (siehe hierzu [GBE 2007]). Eine aktive und optimierte Ausgestaltung der Lieferantenbeziehung kann somit eine wesentliche Hebelwirkung auf das Unternehmensergebnis ausüben.

Erhöhte Effizienz in der Leistungserbringung: Mit der Einführung von DRG wird auch der Druck zunehmen, die durchschnittliche Verweildauer der Patienten zu kürzen. Die Fähigkeit, möglichst rasch und mit geringen Kosten eine Geschäftsbeziehung mit externen Partnern aufbauen zu können, ist eine wesentliche Voraussetzung, um die Effizienz der Leistungs- erbringung noch weiter steigern zu können (vgl. [Gericke et al. 2006, S. 21 f.]).

Steigende Patientenansprüche: Die Ansprüche an die medizinische Leistungserbringung haben aufgrund verschiedenster Faktoren (z. B. zunehmende Mobilität, Wertewandel von der Pflicht- zur Selbstverwirklichungsgesellschaft, technologischer Fortschritt) stark zugenommen. "Medizin soll nicht nur Erkrankungen heilen, sondern zunehmend Gesundheit und Jugendlichkeit bis ins hohe Alter sichern « [Klotz 2003. S. 29]. Um die Qualität der Leistungserbringung auch weiterhin anheben oder zumindest auf dem heutigen Niveau halten zu können, wird die Integration externer Leistungen (i. S. der Besinnung auf die eigenen Kernkompetenzen) an Bedeutung gewinnen. Für die Ausgestaltung der Lieferantenbeziehung wird demnach nicht nur Effizienz, sondern auch Effektivität eine Rolle spielen.

Zunehmende Komplexität in der Beschaffung: Der kontinuierliche Wandel wirtschaftlicher Rahmenbedingungen, der sich z. B. in einer steigenden Bedeutung von Global Sourcing niederschlägt [Stölzle \& Heusler 2003, S. 170], ist auch im Gesundheitswesen zu spüren (bspw. im Generikamarkt). Gerade wegen der Entwicklung von lokalen respektive nationalen Märkten hin zum globalen Markt für Arzneimittel und Medizinprodukte hat die Beschaffung deutlich an Komplexität gewonnen. Dies spiegelt sich beispielsweise an der steigenden Anzahl der zur Verfügung stehenden Produkte wider.

Insgesamt unterscheiden sich die angesprochenen Treiber nicht wesentlich von denjenigen anderer Branchen, jedoch gibt es im Gesundheitswesen weitere Faktoren, die zu einer differenzierten Ausgestaltung der Lieferantenbeziehung (bspw. im Vergleich zur Industrie) führen:

Verkäufermarkt: Das Gesundheitswesen ist im Gegensatz zur Industrie ein klassischer Verkäufermarkt. Der Kunde ist bereit, für das Gut "Gesundheit« i.d.R. jeglichen Preis zu bezahlen (unelastische Preise). Ähnlich sieht es aufseiten 
der Hersteller aus: Da die Krankenhäuser oft vom Verkäufer bestimmter Güter abhängig sind (z. B. aufgrund des Fehlens eines alternativen Produktes und des daraus resultierenden Nachfrageüberhangs), können partnerschaftliche Lieferantenbeziehungen nur sehr schwer aufgebaut werden.

Staatliche Reglementierung und Regulierung: Der Markt für Gesundheit wird vom Staat sowohl fachlich als auch wirtschaftlich vollständig reguliert (z. B. Zulassung, Registrierung und Risikoüberwachung neuer Medikamente) und reglementiert (z.B. Leistungsaufträge öffentlicher Krankenhäuser). Dadurch werden der Handlungsfreiheit der einzelnen Akteure enge Grenzen gesetzt. Neue, innovative Kooperationsformen zwischen Leistungserbringer und Lieferanten (bspw. Parallelimport von Medikamenten) kommen so nur zögerlich zustande. Zudem wirkt sich der hohe Staatsanteil an den Investitionen eher hemmend auf die Innovationsfähigkeit der öffentlichen Krankenhäuser aus, sodass lediglich ein geringer Effektivitäts- bzw. Effizienzdruck vorherrscht (vgl. [Gericke et al. 2006, S. 21]).

Versorgungspflicht im Krisenfall: Per Gesetzgebung besteht für die (öffentlichen) Krankenhäuser eine permanente Versorgungspflicht auch im Krisenfall. Aufgrund der fortschreitenden Globalisierung steht ein Krankenhaus im Krisenfall im Wettbewerb um dieselben knappen Ressourcen mit anderen Krankenhäusern der ganzen Welt. Nur durch eine aktive Beschaffungsplanung und entsprechende Kooperationsvereinbarungen mit Lieferanten können kurzfristig auftauchende Bedarfe gedeckt werden (ein brisantes Beispiel für die unzureichende Lieferkettensteuerung war die im Jahr 2005 aufgrund der befürchteten InfluenzaPandemie durch das Vogelgrippevirus $\mathrm{H}_{5} \mathrm{~N}_{1}$ ausgelöste Übernachfrage nach dem Grippemittel Tamiflu).

Eingeschränkte Handlungsfreiheit in der Sortimentsgestaltung: Ein Krankenhaus ist in seiner Sortimentsgestaltung nicht frei. Aufgrund der Zunahme chronischer Krankheiten (Aller- gien, Rheuma etc.) und der Überalterung der Bevölkerung sind viele Patienten bereits vor dem Eintritt ins Krankenhaus in medizinischer Behandlung. Diese gilt es, auch während des Krankenhausaufenthalts aufrechtzuerhalten. Als Folge davon müssen auch Medikamente und Präparate beschafft werden, die außerhalb des definierten Sortiments liegen.

\section{SRM in Krankenhäusern}

Im Vergleich mit Branchen, in denen das Partner Relationship Management in Forschung und Praxis weit fortgeschritten ist, existieren im Gesundheitswesen heute erste Ansätze. Damit sich SRM in Krankenhäusern auf breiter Front durchsetzen kann, sind praktikable Methoden und Instrumente sowie geeignete Rahmenkonzepte von hoher Bedeutung. Als mögliche Grundlage für die Strukturierung der Thematik bietet sich das Framework von [Eyholzer et al. 2002] an, das drei grundsätzliche Komponenten des SRM unterscheidet (vgl. Abb. 1):

- (Strategisches) Sourcing: Instrumente für die Suche von Informationen bezüglich Lieferanten, Produkten, Konditionen etc., die für die Anbahnung eines Geschäfts erforderlich sind, sowie Instrumente für die Verhandlung bzw. den Vertragsabschluss und für die Bewertung, Entwicklung und Integration der Lieferantenbeziehung.

- (Operatives) Procurement: Instrumente, die die Abwicklung eines Geschäfts unterstützen wie beispielsweise die Bestellübermittlung oder Rechnungsprüfung.

- Monitoring und Controlling: Instrumente für die Messung und Überwachung des strategischen und operativen Einkaufs sowie der Lieferantenleistung und der eingesetzten ITTools.

\subsection{Strategisches Management der Lieferantenbeziehung (Sourcing)}

Aus strategischer Sicht beschäftigt sich das SRM im Kern mit dem Aufbau und der Pflege 

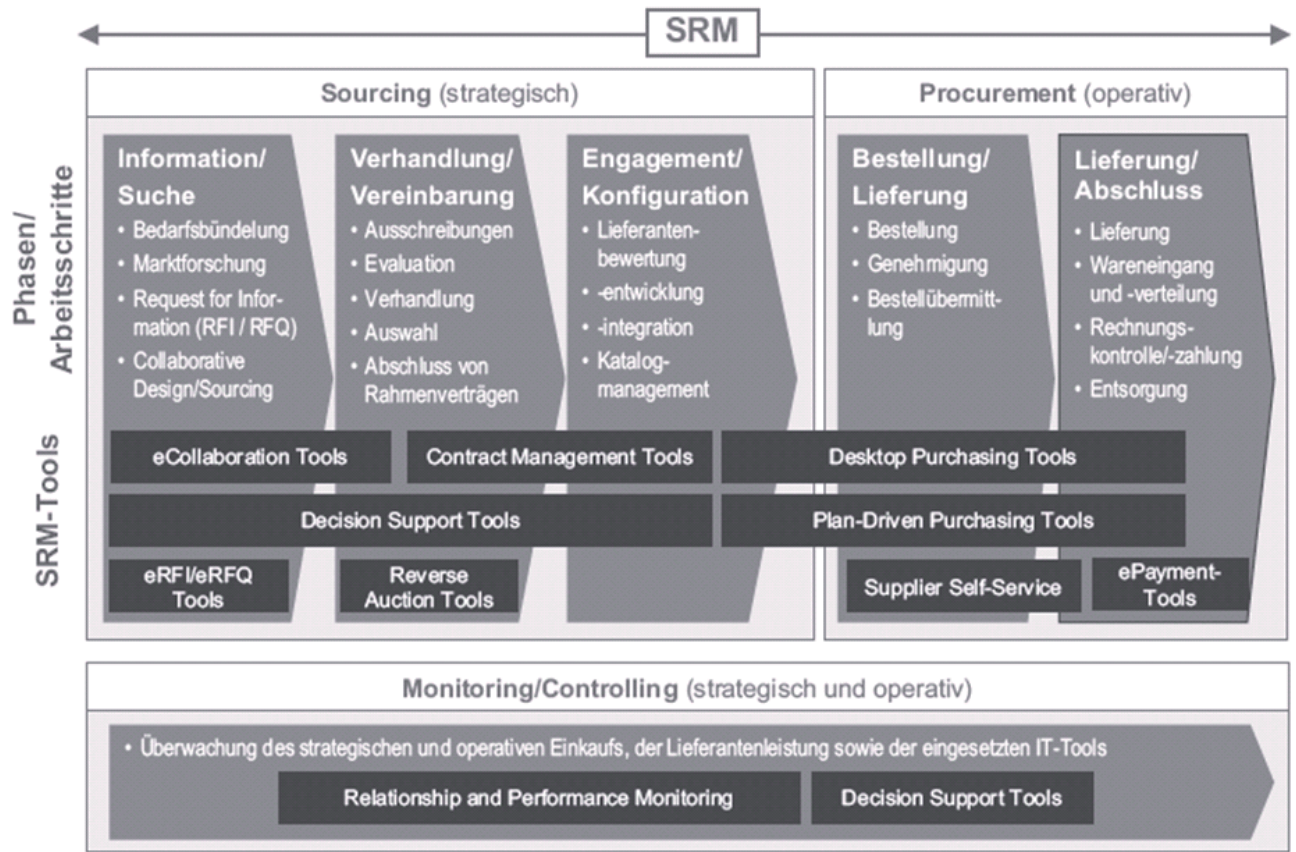

Abb. 1: Komponenten des SRM [Eyholzer et al. 2002, S. 70]

von Lieferantenbeziehungen und mit der Fragestellung, wie stark die externen Partner in das Unternehmen integriert werden sollen: „Dies ist eng verknüpft mit der Frage nach den zur Verfügung stehenden externen, von Lieferanten beziehbaren Ressourcen « [Riemer \& Klein 2002, S. 11]. Diese lassen sich grundsätzlich drei Warengruppen zuordnen (vgl. Abb. 2):

- Patientennahe Güter: Darunter fallen alle Waren, die eine hohe Relevanz für die primäre Leistungserstellung haben, wie beispielsweise Arzneimittel und Medizinprodukte.

- Patientenbezogene Güter: Darunter fallen alle Waren, die eine mittlere Relevanz für die primäre Leistungserstellung haben und von den Patienten direkt wahrgenommen werden (z. B. Lebensmittel, Wäsche).

- Patientenentfernte Güter: Darunter fallen alle Waren, die für die Leistungserstellung zwar gebraucht werden, aber vom Patienten nicht wahrgenommen werden.
Für die Auswahl der Lieferanten spielen gemäß einer Studie des Deutschen Krankenhaus Instituts vor allem der Preis eine wichtige Rolle, aber auch (jedoch weit weniger wichtig) die Produktqualität und die Lieferantenzuverlässigkeit (vgl. [Offermanns 2003, S.49 f.]). Frühere Erfahrungen mit dem Lieferanten, lokale Präsenz, Markenname und die Breite der angebotenen Serviceleistungen werden oftmals als nebensächlich erachtet. Deshalb erstaunt es nicht, dass bei der Mehrheit der Befragten das Ziel der Kosteneinsparung ("den besten Preis erzielen «) immer noch im Vordergrund steht (vgl. [Offermanns 2003, S.47]). Da die kurzfristig orientierten Einkaufshebel für die einzelnen Krankenhäuser (z. B. die durch Verhandlung der Stückpreise realisierbaren Einsparungen) in vielen Warengruppen weitgehend ausgeschöpft sind, wird zukünftig immer mehr die Effektivität einer Lieferantenbeziehung eine Rolle spielen. Um mittel- bis langfristige Einsparungen erzielen zu können, werden deshalb an- 


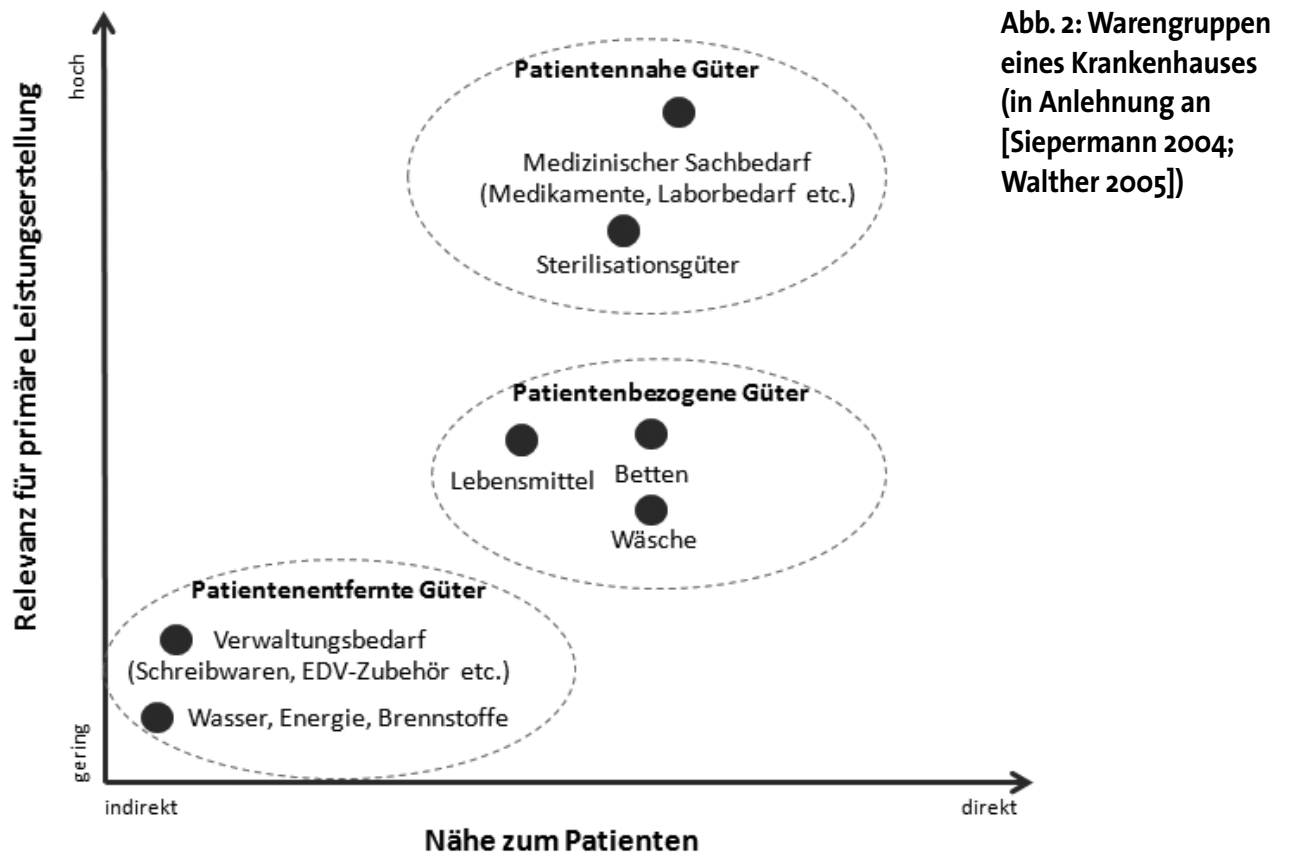

dere Prinzipien wie die Bündelung der Nachfrage in Einkaufskooperationen, standortübergreifendes Category-Management, die Reduzierung der Lieferantenanzahl, die Standardisierung des Produktportfolios oder die Optimierung der gesamten Beschaffungskette an Bedeutung gewinnen (vgl. [Falk \& Da-Cruz 2005, S. 16 f.]).

\subsection{Operative Beschaffungsaufgaben (Procurement)}

Aus operativer Sicht beschäftigt sich das SRM mit sämtlichen erforderlichen Transaktionen, die für die Beschaffungsabwicklung erforderlich sind. Grundsätzlich können diese operativen Teilaufgaben als vorgangsbezogene Teilaufgaben der Beschaffung gesehen werden, die auf den Vorgaben des Sourcings basieren. Während es im (strategischen) Sourcing vor allem um die Effektivität einer Lieferantenbeziehung geht, zielt das (operative) Procurement auf eine höchstmögliche Effizienz ab. Dabei kommt der IT-gestützten Abwicklung der klassischen operativen Beschaffungsaufgaben (E-Procurement) besondere Beachtung zu. Einer aktuellen Umfrage des Bundesverbandes Medizintechnologie zufolge sehen Krankenhäuser den Nutzen von E-Procurement vor allem in der Optimierung der Beschaffungsprozesse, in Kosteneinsparungen und in der Versorgungssicherheit (vgl. [BVMed 2007]). Weitere Vorteile wurden in der Zeitersparnis bei der Bestellabwicklung, im Wegfall manueller Vorgänge und in der Verringerung von Fehllieferungen gesehen. Zwei Drittel der Befragten haben angegeben, bereits eine elektronische Schnittstelle zu Plattformen eingerichtet zu haben, über die elektronische Bestellungen abgewickelt werden. Einen elektronischen Lieferschein haben $38 \%$, eine elektronische Rechnung $35 \%$ realisiert. Weitaus weniger verbreitet sind Lösungen für die Faxautomation oder die Vernetzung mit Geschäftspartnern mittels EDI (Electronic Data Interchange). Als wesentliches Hindernis für eine breitere Verankerung von E-Procurement im Gesundheitswesen wurden die zu hohen Kosten der elektronischen Plattformen gesehen. Dennoch erwarten 35\% 
der Krankenhäuser den Durchbruch von E-Procurement innerhalb der nächsten drei Jahre. Im Vergleich zur Automobil- und Luftfahrtbranche, wo bereits $85 \%$ der Unternehmen SRM-Systeme aktiv nutzen, ist das verbleibende Potenzial hoch (vgl. [BME 2006]).

\subsection{Monitoring und Controlling der Lieferantenbeziehung}

Neben den genannten strategischen und operativen Aufgaben, die das SRM als Teil eines umfassenden Partner Relationship Management zu leisten hat, ist auch eine konsequente Überwachung der Leistungen des Lieferanten, aber auch des innerbetrieblichen Einkaufs notwendig. Dabei stellen die laufend zu erfassenden Informationen bezüglich Mengen, Preisen, Zeiten und Qualitäten die Eingangsgrößen des Supplier Performance Measurement dar. Insbesondere durch die Erhebung von Qualitätsmerkmalen lässt sich die Lieferanten-Performance nicht nur aus wirtschaftlicher Sicht beurteilen, sondern auch über Faktoren wie den Anteil der akzeptierten und rechtzeitigen Lieferungen (Zuverlässigkeit), Know-how und Güte der erbrachten Dienstleistungen. Gerade in diesem Bereich herrscht heute bei den Krankenhäusern großer Handlungsbedarf (nach Angaben von [Bucher 2007; Fischer \& Maag 2007]).

\section{SRM im Universitätsspital Zürich - eine Fallstudie}

Die breite Einführung eines umfassenden und ganzheitlichen SRM steht in vielen Krankenhäusern noch aus. Die vorliegende Fallstudie stellt einen ersten Erfahrungsbericht dar, aus dem sich allerdings bereits Potenziale ableiten lassen, die durch die Einführung verschiedener Instrumente des SRM realisiert werden können.

\subsection{Strategisches Sourcing: Die Gründung des Einkaufsverbunds Symbia}

Mit dem Ziel der Realisierung von Einsparungsund Kostensenkungspotenzialen sowie der
Nutzung von Synergien in beschaffungsrelevanten Aufgabenstellungen haben sich im Jahr 2006 vier Krankenhäuser (darunter auch das Universitätsspital Zürich) zusammengefunden, um einen Einkaufsverbund zu gründen (vgl. [Symbia 2007]). Beim Zusammenschluss wurde vorerst bewusst eine überschaubare Anzahl beteiligter Partner ausgesucht, um in der intensiven Anfangs- und Aufbauphase rasch die gemeinsame Strategie umsetzen zu können. Aufgrund des Fokus der gemeinsamen Strategie stand von Anfang an fest, dass für einen nachhaltigen Erfolg von Symbia nicht nur die Produktpreise optimiert werden sollen, sondern auch die Prozesskosten, die entlang der gesamten Wertschöpfungskette anfallen. Um die Pragmatik auch bei der Umsetzung beizubehalten, entschloss man sich in einer ersten Phase dazu, lediglich den Einkauf und die Beschaffungslogistik von Medizinprodukten zu optimieren. Um die Nachfrage der vier Krankenhäuser überhaupt bündeln zu können, war es notwendig, Transparenz des Produktsortiments bzw. Produktkatalogs durch Kompatibilität der einzelnen Datenbestände (eine gemeinsame Sprache) sicherzustellen. Daher entschied man sich für die Nutzung von Tools der Medical Columbus AG, die u.a. eine Referenzdatenbank mit über 2 Millionen normierten Medizinprodukten unterhält. Durch ein Mapping der Produktinformationen der einzelnen Krankenhäuser mit der Referenzdatenbank konnten die ursprünglich heterogenen Stammdaten durch eine einheitliche und neutrale Artikelbezeichnung erweitert werden. Dies führte nicht nur zu mehr Transparenz innerhalb des Einkaufverbunds, da dadurch Preise und Konditionen vergleichbar wurden, sondern war auch eine wesentliche Voraussetzung, um die Bestelltransaktionen künftig elektronisch abwickeln zu können. Ziel ist es, mittels Standardschnittstelle möglichst alle Lieferanten an die Transaktionsplattform von Medical Columbus anzuschließen (vgl. Abb. 3). 


\subsection{Operatives Procurement: Die Nutzung von Desktop Purchasing}

Eine weitere Herausforderung für die Einkaufsabteilung des Universitätsspitals Zürich lag in der Abwicklung des operativen Procurements. Dessen Optimierung hatte das Universitätsspital Zürich bereits vor der Etablierung des Einkaufsverbunds durch ein Projekt zur Effizienzund Effektivitätssteigerung des operativen Procurements initiiert. Da die benötigten Artikel bis jetzt ausschließlich papierbasiert von den Bedarfsträgern bestellt wurden, lief der Beschaffungsprozess weitestgehend manuell $a b$. Dadurch wurden umfassende Kontroll- und Steuerungsmöglichkeiten erschwert.

Durch die Einführung einer SRM-Plattform wurde ein geeignetes Werkzeug implementiert, das eine elektronische Integration von der Bestellaufnahme auf den Stationen über die Verarbeitung in der Einkaufs- und Logistikabteilung bis hin zur Bestellauslösung beim
Lieferanten ermöglichte. Als praktischste Lösung für die Bestellaufnahme erwies sich hierbei die Einführung eines katalogbasierten, elektronischen Bestellsystems (SAP/SRM), das mittels Stationsterminals auf dem Intranet abzurufen ist (vgl. Abb. 3). Im Zuge dieses Projekts wurden wichtige Voraussetzungen für die Bildung des Einkaufsverbunds geschaffen, unter anderem die Verfügbarkeit einer vollständigen und zentral zu bewirtschaftenden Artikeldatenbank sowie qualitativ hochwertige Bestellinputs. Heute kann durch die Kopplung von SAP/SRM mit der Artikeldatenbank auch auf dem Bestellsystem die einheitliche und neutrale Artikelbezeichnung genutzt werden.

\subsection{Nutzen für das Universitätsspital Zürich}

Die Umsetzung der oben gezeigten Maßnahmen in den Bereichen strategisches Sourcing und operatives Procurement erwies sich sowohl für die Einkaufs- und Logistikabteilung als auch

\section{Strategisches Sourcing}

(Einkaufsverbund)

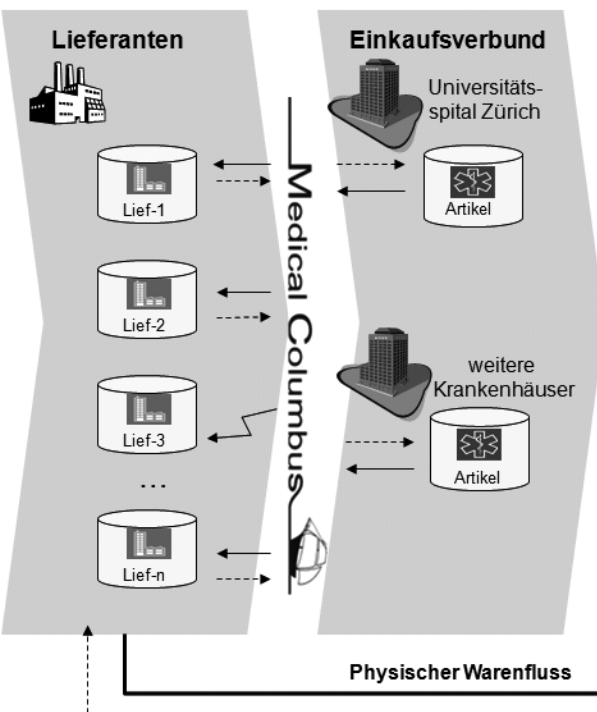

Operatives Procurement (einzelnes Krankenhaus)

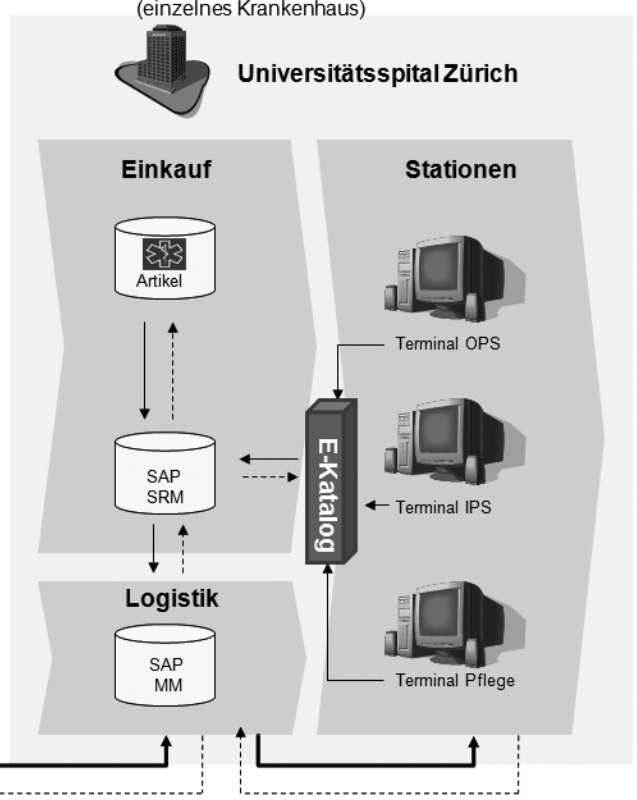

Abb. 3: SRM-Tools im Universitätsspital Zürich 
für die Pflege des Universitätsspitals als äußerst nützlich. Für das Pflegepersonal auf den Stationen ergab die Lösung folgende Vorteile (nach Angaben von [Fischer \& Maag 2007]):

- Die Bestellungen konnten mittels Webbrowser (mit gewohnter Oberfläche) und innerhalb nur einer Anwendung getätigt werden. Dadurch reduzierte sich der Aufwand für Schulungen auf ein Minimum.

- Die Informationsbasis für die Bestellaufnahme wurde erheblich verbessert (mehr Informationen zu Produkteigenschaften, aktuellere Preise, Lieferstatus etc.). Außerdem wurde die Anzahl manueller Bestellungen auf ein Minimum reduziert; nach der Einführung des integrierten SRM-Systems liegt der Anteil elektronischer Bestellungen bei über $80 \%$ und ist stetig steigend.

- Die Suche nach bestimmten Produkten wurde durch den vollständigen Produktkatalog erleichtert.

Für die Einkaufs- und die Logistikabteilung ergab die Lösung folgende Vorteile:

- Durch den Zusammenschluss des Universitätsspitals mit einem Einkaufsverbund und durch die Transparenz des Produktsortiments sowie die Kompatibilität des Produktkatalogs mit den Partner-Institutionen wurden Preise und Konditionen vergleichbar. Diese können als Verhandlungsbasis mit Lieferanten genutzt werden.

- Dank der Implementierung einer Schnittstelle zum Materialwirtschaftssystem ließ sich der gesamte Prozess von der Bedarfsermittlung bis zur Auslieferung auf den Stationen wesentlich beschleunigen, und Medienbrüche konnten minimiert werden.

- Außerdem wurde durch die breitere Informationsbasis und Minimierung der Medienbrüche neues Potenzial für das Sourcing (z. B. Pooling von Bedarfsmeldungen), aber auch für das Monitoring und Controlling der Lieferantenbeziehungen geschaffen.

\section{Mehr SRM im Gesundheitswesen}

Obwohl heute kurzfristig motivierte Kosteneinsparungen oft die Gründe für die Einführung von SRM in den Krankenhäusern darstellen, verbindet SRM neben der effizienten Abwicklung traditioneller operativer Tätigkeiten des Einkaufs (z. B. E-Procurement) insbesondere auch strategische Aspekte der Beschaffung, die auf die Effektivität der Lieferantenbeziehung abzielen. Diese werden für einen nachhaltigen Erfolg zunehmend an Bedeutung gewinnen. Im Vergleich zur Automobil- und Luftfahrtindustrie, wo bereits $85 \%$ der Unternehmen SRM eingeführt haben [BME 2006], steckt das Gesundheitswesen noch in den Anfängen. Dennoch lassen sich erste SRM-Konzepte, wie die Fallstudie zeigt, durch eine pragmatische Anfangsstrategie und vergleichsweise einfache organisatorische und technische Maßnahmen im Krankenhausumfeld relativ schnell umsetzen. Die Gründung oder der Beitritt zu einem Einkaufsverbund ist ein erster Schritt in diese Richtung. Durch (virtuelle) Zusammenlegung der Verbräuche entsteht Transparenz, die bei Verhandlungen über Preise und Konditionen genutzt werden kann und somit augenblicklich zu Quick Wins im Einkauf führt. Gleichwohl können aus der engeren Zusammenarbeit mit anderen Krankenhäusern, aber auch mit externen Partnern weit mehr Potenziale ausgeschöpft werden als nur bessere Preise bei der Beschaffung. Der große Nutzen entsteht vor allem durch die Kombination von Einkauf und Logistik der Partner-Institutionen (bspw. Optimierung der Lagerbewirtschaftung, Cross Docking, Zusammenlegung der physischen Bestände oder gemeinsame Nutzung der Infrastruktur). Prozessintegration entlang der Wertschöpfungskette wird für eine ganzheitliche und nachhaltige Optimierung der logistischen Prozesse eines Krankenhauses zentral werden. Das Supplier Relationship Management kann im Kontext der wertekettenbezogenen Managementkonzepte sicherlich seinen Beitrag dazu leisten. Damit 
sich SRM im Gesundheitswesen auf breiter Front durchsetzen kann, sind von Forschung und Praxis deshalb praktikable Methoden und Instrumente gefragt.

\section{Literatur}

[Albrecht \& Töpfer 2006] Albrecht, M.; Töpfer, A.: Erfolgreiches Changemanagement im Krankenhaus - 15 Punkte Sofortprogramm für Kliniken. Springer-Verlag, Heidelberg, 2006.

[BME 2006] Bundesverband Materialwirtschaft, Einkauf und Logistik e.V. (Hrsg.): Marktstudie 2006: Supplier Relationship Management, 2006, www.bme.de, Zugriff am 25.08.2007.

[Bucher 2007] Interview mit R. Bucher, Leiter Einkauf \& Versorgung des Kantonsspitals Aarau, Schweiz, am 13.08.2007.

[BVMed 2007] Bundesverband Medizintechnologie e.V. (Hrsg.): BVMed-Umfrage: Elektronisches Beschaffungswesen im Gesundheitsmarkt vor dem Durchbruch, 2007, www.bvmed.de/presse/ pressemitteilung/BVMed-Umfrage_Elektronisches Beschaffungswesen_im_Gesundheitsmarkt_vor_ dem_Durchbruch.html, Zugriff am 25.08.2007.

[Eyholzer et al. 2002] Eyholzer, K.; Kuhlmann, W.; Münger, T.: Wirtschaftlichkeitsaspekte eines partnerschaftlichen Lieferantenmanagements. In: HMD - Praxis der Wirtschaftsinformatik, 39. Jg., Heft 228, 2002, S. 66-76.

[Falk \& Da-Cruz 2005] Falk, K.; Da-Cruz, P.: Balance Scorecard in der Krankenhausbeschaffung. Baumann Fachverlage, Kulmbach, 2005.

[Fischer \& Maag 2007] Interview mit E. Fischer \& D. Maag, Bereichsleiter Einkauf \& Logistik des Universitätsspitals Zürich, Schweiz, am 05.07.2007.

[Flintrop 2006] Flintrop, J.: Auswirkungen der DRGEinführung: Die ökonomische Logik wird zum Maß der Dinge. In: Deutsches Ärzteblatt, 103. Jg., 2006, Heft 46, S. A-3082 / B-2683 / C-2574.

[GBE 2007] Gesundheitsberichterstattung des Bundes (Hrsg.): Kosten je Krankenhaus, 2007, www. gbe-bund.deloowag21-install/servlet/oowa/aw92/ dboowasys921.xwdevkit/xwd_init?gbe.isgbetol/ $x s$ start_neu/376788313/62993031, Zugriff am 25.08 .2007 .

[Gericke et al. 2006] Gericke, A.; Rohner, P.; Winter, R.: Vernetzungsfähigkeit im Gesundheitswesen - Notwendigkeit, Bewertung und systemati- sche Entwicklung als Voraussetzung zur Erhöhung der Wirtschaftlichkeit administrativer Prozesse. In: HMD - Praxis der Wirtschaftsinformatik, 43. Jg., Heft 251, 2006, S. 20-30.

[Klotz 2003] Klotz, T.: Lifestyle-Medizin und Rationierung - Was bringt die Zukunft? In: Blickpunkt der Mann - Wissenschaftliches Journal für Männergesundheit, 1. Jg., 2003, Heft 1, S. 29-31.

[Offermanns 2003] Offermanns, M.: Krankenhaus Barometer 2003, Deutsches Krankenhaus Institut, Düsseldorf, 2003.

[Riemer \& Klein 2002] Riemer, K.; Klein, S.: Supplier Relationship Management. In: HMD - Praxis der Wirtschaftsinformatik, 39. Jg., Heft 228, 2002, S. 5-22.

[Siepermann 2004] Siepermann, C.: Stand und Entwicklungstendenzen der Krankenhauslogistik in Deutschland. Verlag für Wissenschaft und Forschung, Berlin, 2004.

[Stölzle \& Heusler 2003] Stölzle, W.; Heusler, K.: Supplier Relationship Management - Entstehung, Konzeptverständnis und methodisch-instrumentelle Anwendung. In: Bogaschewsky, R.; Götze, U. (Hrsg.): Management und Controlling von Einkauf und Logistik. Gernsbach, 2003, S. 167-194.

[Symbia 2007] Symbia (Hrsg.): Informationen zum Einkaufsverbund Symbia unter www.symbia.ch, Zugriff am 25.08.2007.

[Walther 2005] Walther, M.: Auf der Suche nach operativer Exzellenz im Krankenhaus: Logistik als Rationalisierungsinstrument und strategischer Wettbewerbsfaktor in einem dynamischen Marktumfeld. Friedrich-Alexander-Universität Erlangen-Nürnberg, 2005.

lic. oec. HSG Tobias Mettler

Dr. oec. HSG Peter Rohner

Universität St. Gallen Institut für Wirtschaftsinformatik Kompetenzzentrum Health Network Engineering Müller-Friedberg-Str. 8

$\mathrm{CH}-9000$ St. Gallen \{tobias.mettler, peter.rohner\}@unisg.ch http://ehealth.iwi.unisg.ch 